\title{
A Novel Mobile Acoustic Uroflowmetry: Comparison With Contemporary Uroflowmetry
}

\author{
Young Ju Lee ${ }^{1,2}$, Michelle M. Kim³ , Sang Hun Song ${ }^{1}$, Sangchul Lee ${ }^{1}$ \\ ${ }^{1}$ Department of Urology, Seoul National University Bundang Hospital, Seongnam, Korea \\ ${ }^{2}$ Department of Urology, CHA Ilsan Medical Center, CHA University, Goyang, Korea \\ ${ }^{3}$ Department of Urology, Massachusetts General Hospital, Boston, MA, USA
}

Purpose: This study aimed to evaluate the accuracy and reliability of a new smartphone-based acoustic uroflowmetry compared to conventional uroflowmetry.

Methods: This was a prospective validation study enrolling 128 subjects from September 2017 to April 2018 comparing a novel acoustic uroflowmetry to conventional uroflowmetry in an outpatient urologic clinic at Seoul National University Bundang Hospital. Visual comparison of flow patterns and uroflow parameters such as maximum flow rate (Qmax), average flow rate (Qavg), and voided volume were compared between the 2 techniques. Reliability and accuracy of the uroflowmetry results were compared using Pearson correlation coefficient (PCC) and Student t-test, respectively.

Results: One hundred twelve participants were included in the study. Of these, 77 had baseline urologic comorbidities while 35 were normal participants. Flow patterns between the 2 uroflowmetry techniques demonstrated strong visual correlation. When compared to conventional uroflowmetry, all 3 parameters of voiding in male participants showed a very robust correlation with PCC of $0.88,0.91$, and 0.95 for Qmax, Qavg, and voided volume, respectively. Among female participants, we observed a PCC of $0.78,0.93$, and 0.96 for Qmax, Qavg, and voided volume, respectively. The Qmax showed a statistically significant difference in both sexes between the 2 methods, although the absolute value was small.

Conclusions: Uroflowmetry using acoustic analysis demonstrates comparable findings to conventional uroflowmetry. This provides an opportunity to perform uroflowmetry in the clinic or at home in a reliable, inexpensive manner. Future large-scale prospective studies are required to further validate our results.

Keywords: Acoustic uroflowmetry; Mobile; Smartphone

- Fund/Grant Support: This work was supported by grant No S2534278 from the Ministry of SMEs and Start-Ups Research Fund. Technical support was made by Soundable Health, Inc.

- Research Ethics: This study was approved by the Institutional Review Board of Seoul National University Bundang Hospital (B-1912/585-301).

- Conflict of Interest: The author of this publication (S. Lee) holds minimal stock ownership in Soundable Health, Inc. The terms of this arrangement have been reviewed and approved by the Seoul National University Bundang Hospital in accordance with its policy on objectivity in research. Except for that, no potential conflict of interest relevant to this article was reported.

\section{INTRODUCTION}

Uroflowmetry is a noninvasive measurement of excreted urine volume over time. It allows measurement of voided volume, voiding time, average flow rate (Qavg), and maximum flow rate (Qmax), as well as abnormal flow patterns [1]. Current uroflowmetry is an office-based procedure designed to measure characteristics of urine excretion while maintaining privacy.
Corresponding author: Sangchul Lee (iD https://orcid.org/0000-0002-3665-8336 Department of Urology, Seoul National University Bundang Hospital, Seoul National University College of Medicine, 82 Gumi-ro 173beon-gil, Bundang-gu, Seongnam 13620, Korea

Email: slee@snubh.org

Submitted: July 16, 2020 / Accepted after revision: August 25, 2020 
However, some patients have difficulty with voiding in an unfamiliar environment, or due to an inconvenient or unnatural timing of voiding, which may require repeated tests for accurate data [2]. The need for a comfortable, convenient, and even portable, home-based device has led to the development of novel uroflowmetry technology. These advancements include soundbased uroflowmetry, optical uroflowmetry, and video-based voiding devices [3-6].

On performing uroflowmetry, the patient voids into a uroflowmeter in a private setting, ideally with a normal to strong desire to void [7]. If an abnormal void occurs, repeated assessment is necessary to try to reproduce normal behavior. Therefore, accuracy in uroflowmetry requires appropriate time, space, and privacy which may be difficult in the office setting. In contrast to previous methods, acoustic uroflowmetry can be done regardless of time and spatial limitations with a smartphone in the home setting rather than using an office-based uroflowmeter. The true value of this method lies in its versatility and convenience.

Traditionally, urine flow rates are calculated by a weight transducer, spinning disc, or change in capacitance. Sound can be used to calculate urine flow by analyzing the acoustic change between air-water interfaces caused by contact of the urine stream with the surface of the toilet water. In 2009, Hitt et al. [3] used sound to measure the urinary flow, demonstrating the possibility of correlating acoustic and flow parameters. In 2011, Zvarova et al. [4] also reported the results of sonouroflowmetry. In their initial feasibility study, they found similar flow curves among men, despite subtle differences in the voiding time and flow rates compared to standard uroflowmetry $[8,9]$.

We tested a new sound-based uroflowmetry technology to describe and predict the urine flow rate by recording the sound of urination using a smartphone. Accuracy and reliability of the results were compared to the results of conventional uroflowmetry.

\section{MATERIALS AND METHODS}

\section{Patient Selection}

Patients were recruited from Seoul National University Bundang Hospital from September 2017 to April 2018. This included patients aged 20 or more, both with and without urologic comorbidities. All participants gave consent for use of both the acoustic and conventional uroflowmetry in the clinic. Exclusion criteria included illiteracy, poor sound quality including an un- expected sudden noise such as a phone ringing, groans, conversations generated during each uroflowmetry session, voided volume $<20 \mathrm{~mL}$, or people who could not complete the uroflowmetry. Males voiding in a sitting position were excluded because of the difference in sound quality. This study was approved by the Institutional Review Board at Seoul National University Bundang Hospital (B-1912/585-301).

\section{Acoustic Uroflowmetry System}

The acoustic uroflowmetry system uses a wireless, smartphonebased approach with a recording function to analyze urinary flow. The sound data was recorded in real-time with a smartphone application. Several parameters were calculated based on the sounds collected including voided volume, urinary flow parameters (e.g., Qmax and Qavg), urinary flow patterns (e.g., continuous, intermittent), and time-related parameters (e.g., time to maximal void, duration of void). Sound features were analyzed through audio processing, signal preprocessing, and spectrum analysis. Prediction models were applied to calculate urine flow and parameters. After postprocessing, the data for accuracy, voiding parameters of uroflowmetry were generated.

Recorded sound was analyzed by audio editing programs (Audacity by Audacity Open Source Team, GoldWave by GoldWave Inc.). Pre- and postprocessing of sound signals and development of flow prediction models were conducted with MATLAB (MathWorks, Inc., Natick, MA, USA) and python (Python Software Foundation, Wilmington, DE, USA). In addition to the sound analysis algorithm, pre- and postprocessing refinement to enhance accuracy was added to remove short-term artifacts and outliers, to calibrate background noise levels and to remove specific noise bands. Differences in urogenital anatomy of men and women as well as their posture during urination were found to affect the turbulent characteristics of urine flow and sound events generated by urination. Two separate prediction models were developed and applied for each sex (Fig. 1).

\section{Urinary Flow Measurement in a Laboratory Setting}

A laboratory setting was made in a typical bathroom to measure the urinary flow with a precision scale while recording the sound of urination using smartphones $1 \mathrm{~m}$ away from the toilet (Fig. 2). An actual toilet was set on a precision scale and the details of precision scale were transmitted and analyzed on a computer. A standard, daily bathroom environment was used to reproduce the acoustic characteristics of background and external noise similar to ambient noise experienced in the office. 

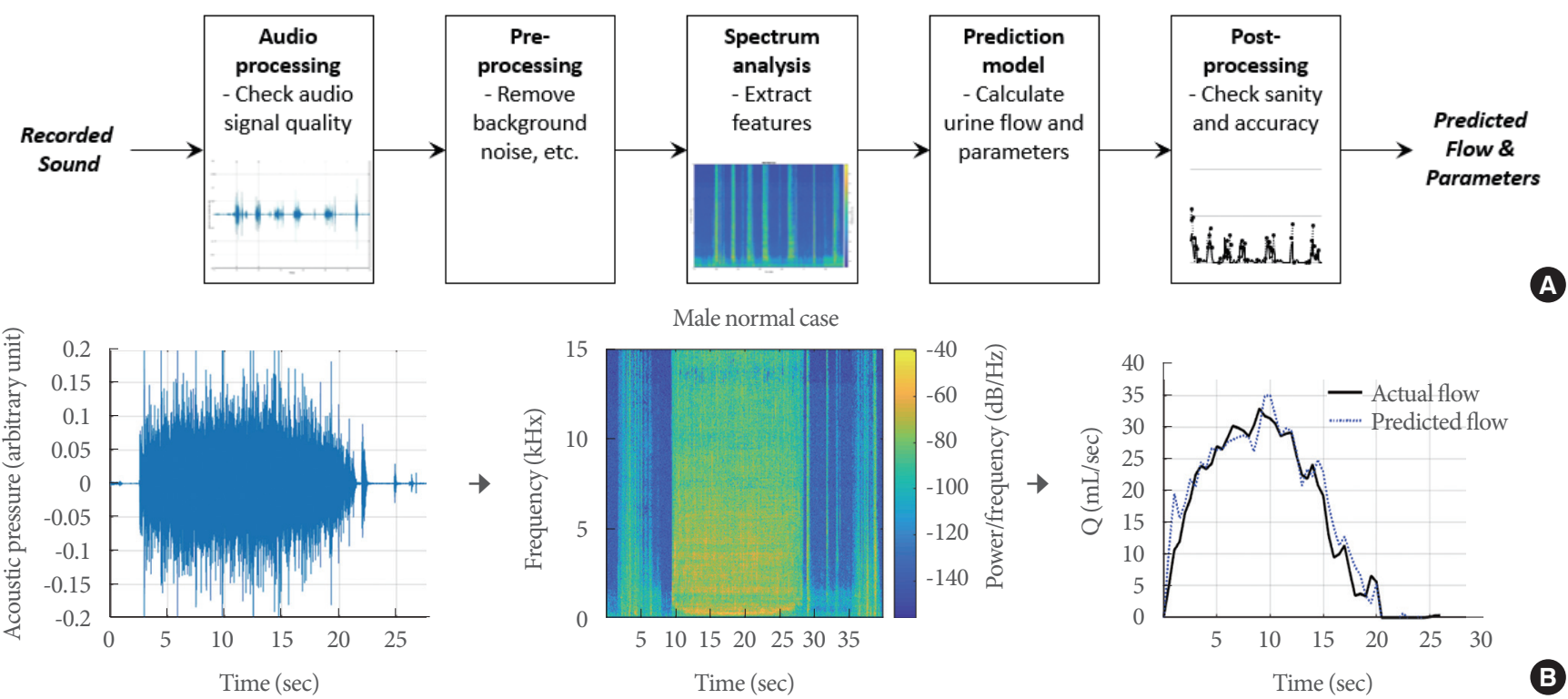

Male normal case
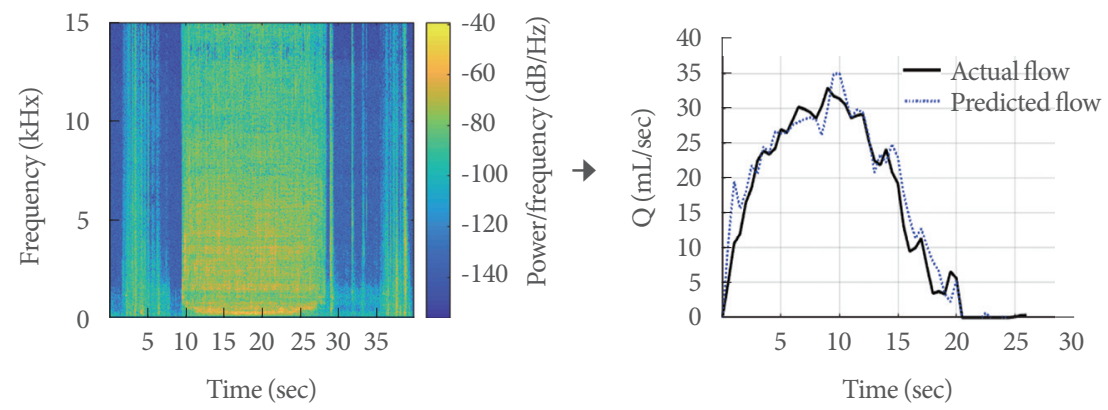

B

Male abnormal case
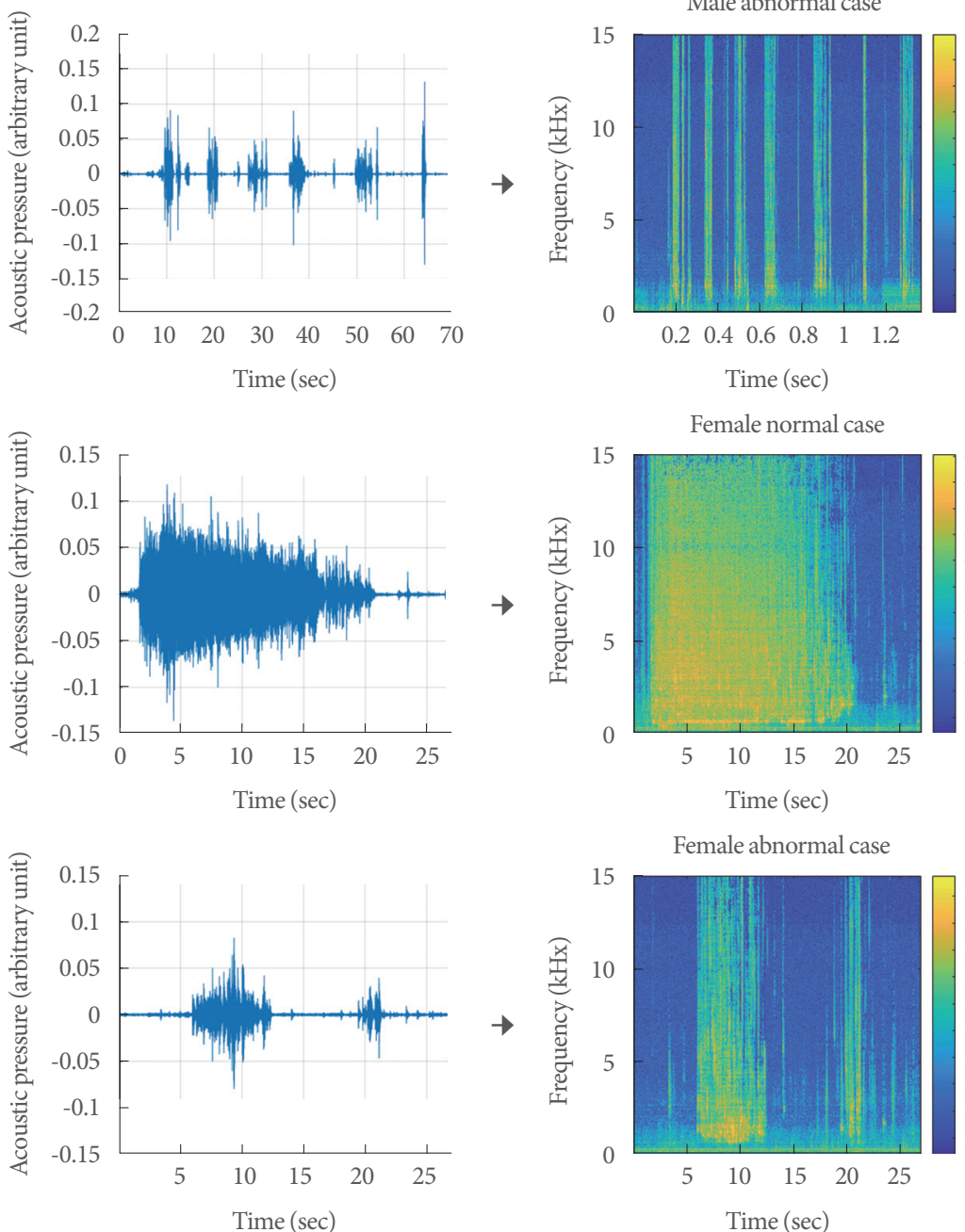

Time (sec)

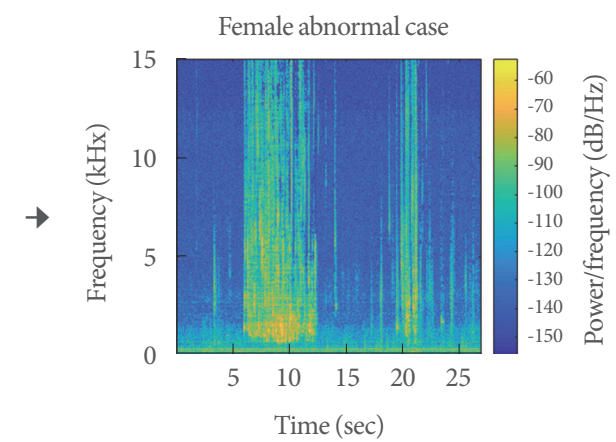

Female normal case
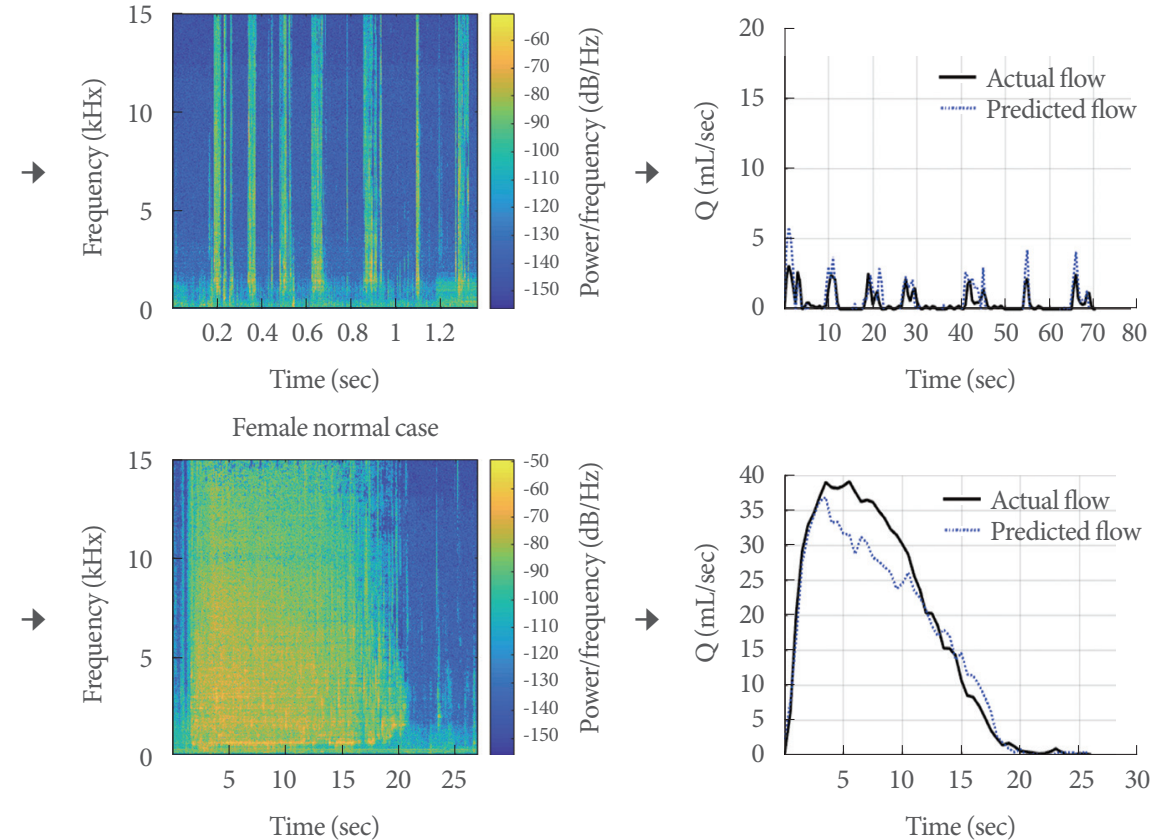

C

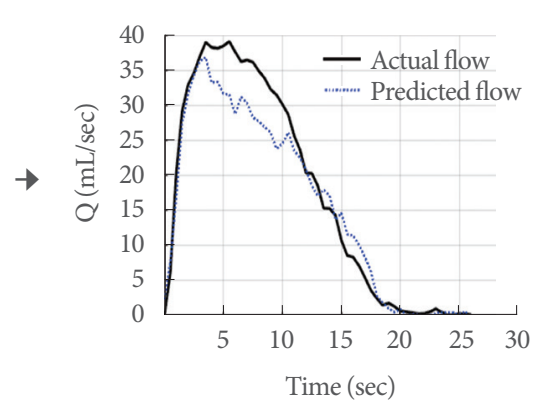

D
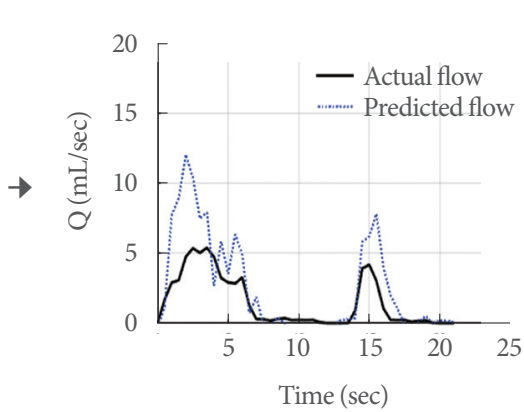

E

Fig. 1. Acoustic uroflowmetry system and examples of male and female uroflowmetry. (A) Flow sheet of sound analysis process. (B, C) Examples of male acoustic uroflowmetry. (D, E) Examples of female acoustic uroflowmetry. 


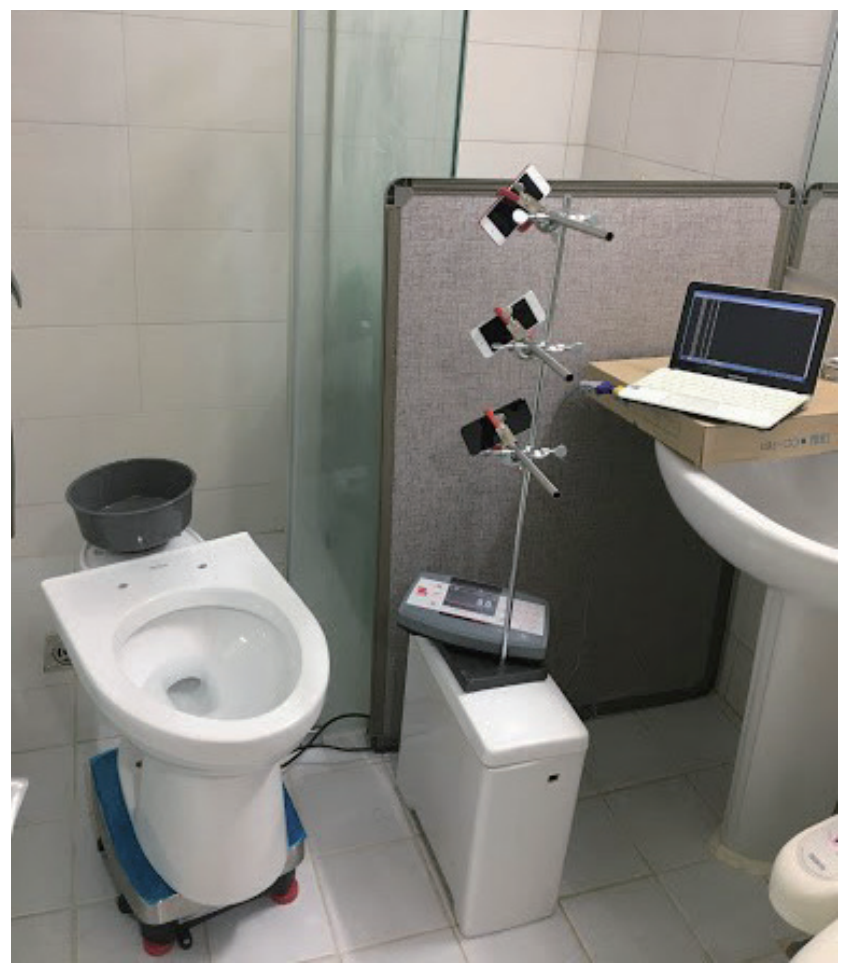

Fig. 2. A laboratory setting to measure the urinary flow while recording urination sound.

\section{Prediction Model}

Forty-eight male and 28 female patients were recruited for development of the initial prediction model. Male patients had a mean age of 42.5 years (range, 22-83 years) and voided 242 times. The average voided volume was $290.0 \mathrm{~mL}$ (range, 34.4$868.3 \mathrm{~mL}$ ) and the average acoustic predicted voided volume was $303.9 \mathrm{~mL}$ (range, 36.3-982.4 mL). The correlation between voided volume and predicted voided volume was excellent (Pearson correlation coefficient $[\mathrm{PCC}]=0.96$ ). Average Qmax and predicted Qmax were $24.0 \mathrm{~mL} / \mathrm{sec}$ (range, 3.3-43.3 mL/ $\mathrm{sec}$ ) and $25.4 \mathrm{~mL} / \mathrm{sec}$ (range, $5.5-43.1 \mathrm{~mL} / \mathrm{sec}$ ), respectively. The correlation was very robust $(\mathrm{PCC}=0.85)$.

Measurements were repeated for female subjects in the same setting. Twenty-eight females had a mean age of 44.8 years (range, 21-77 years) and voided 83 times. Average voided volume was $220.3 \mathrm{~mL}$ (range, 31.4-573.4 mL) and the average acoustic predicted voided volume was $221.6 \mathrm{~mL}$ (range, 53.0$654.1 \mathrm{~mL})$. The correlation was excellent $(\mathrm{PCC}=0.93)$. Average Qmax and predicted Qmax was $24.7 \mathrm{~mL} / \mathrm{sec}$ (range, 5.5-61.7 $\mathrm{mL} / \mathrm{sec}$ ) and $24.8 \mathrm{~mL} / \mathrm{sec}$ (range, $12.0-58.8 \mathrm{~mL} / \mathrm{sec}$ ), respectively. The correlation was robust $(\mathrm{PCC}=0.78)$.

\section{Comparative Testing With Conventional Uroflowmetry}

After establishing the initial prediction model with the urinary flow measurements in a laboratory setting, patients and normal participants were recruited for the pilot and clinical test. All the participants were required to perform the conventional uroflowmetry (CubeFlow_S, MCube technology, Seoul, Korea) while also recording their urination using a smartphone located about $1 \mathrm{~m}$ away from the uroflowmeter and the participant.

\section{Statistical Analysis}

We compared the 2 uroflowmetry tests by calculating the means and standard deviations for each parameter. Correlation was calculated by PCC for Qmax, Qavg, and voided volume. Student t-test was performed to compare each of the urine flow parameters. Python 3.6.9, R ver. 3.4.0 (R Foundation for Statistical Computing, Vienna, Austria), and Excel (Microsoft, Redmond, WA, USA) were used for the statistical analysis of the prediction model. $\mathrm{P}<0.05$ was considered to be statistically significant.

After the pilot study with patients, a sample size for normal participants was estimated with a power of $0.9,5 \%$ type 1 error, and noninferiority margin of 0.34 . Assuming a $25 \%$ of dropout rate, incomplete study, or sound quality problems, 18 males and 17 females were recruited as volunteers.

\section{RESULTS}

A total of 72 men and 56 women were recruited from September 2017 to April 2018. After excluding those who had small voided volume $(n=3)$, failure to void $(n=3)$, low quality of the sound ( $\mathrm{n}=10)$, a total of 66 men (48 patients, 18 normal participants), and 46 women (29 patients, 17 normal participants) were included in the analysis. Table 1 shows the patient characteristics including voiding parameters from the uroflowmetry. Sound generated by urination differs by sex, likely due to differences in the anatomy and posture during urination. Males produce louder sounds during urination due to the standing position, which has no barrier for sound diminutions. Therefore, separate models were developed for each sex and applied accordingly.

All flow patterns were compared visually by a single experienced urologist. Flow patterns between the 2 uroflowmetry methods showed a strong visual correlation (Fig. 3). A few normal female participants showed significantly lower predicted Qmax when compared with the conventional uroflowmetry. 
Table 1. Summary of the conventional uroflowmetry of participants

\begin{tabular}{|c|c|c|c|c|}
\hline \multirow{3}{*}{ Variable } & \multicolumn{4}{|c|}{ Participant type } \\
\hline & \multicolumn{2}{|c|}{ Patients } & \multicolumn{2}{|c|}{ Normal participants } \\
\hline & Male $(\mathrm{n}=48)$ & Female $(\mathrm{n}=29)$ & Male $(\mathrm{n}=18)$ & Female $(n=17)$ \\
\hline Age (yr) & 65.9 & 66.5 & 34.6 & 31.1 \\
\hline Voided volume (mL) & 175.1 & 188.6 & 273.6 & 310.1 \\
\hline Voiding time (sec) & 25.2 & 29.7 & 15.4 & 13.7 \\
\hline Maximum flow rate $(\mathrm{mL} / \mathrm{sec})$ & 13.1 & 17.6 & 32.7 & 38.4 \\
\hline Average flow rate (mL/sec) & 6.8 & 8.8 & 15.9 & 20.8 \\
\hline
\end{tabular}

Values are presented as mean.
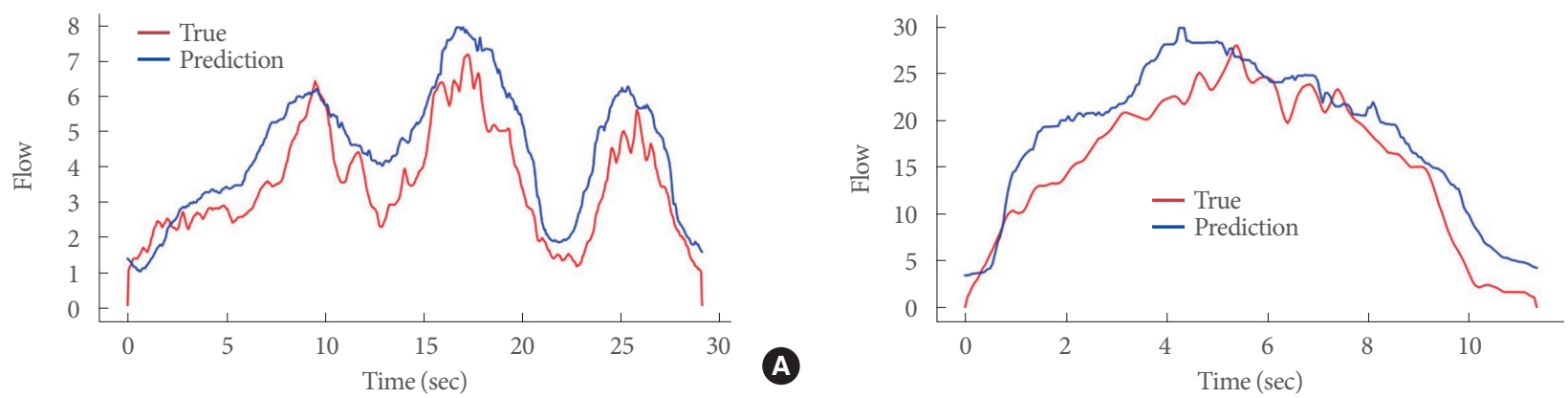

(D)
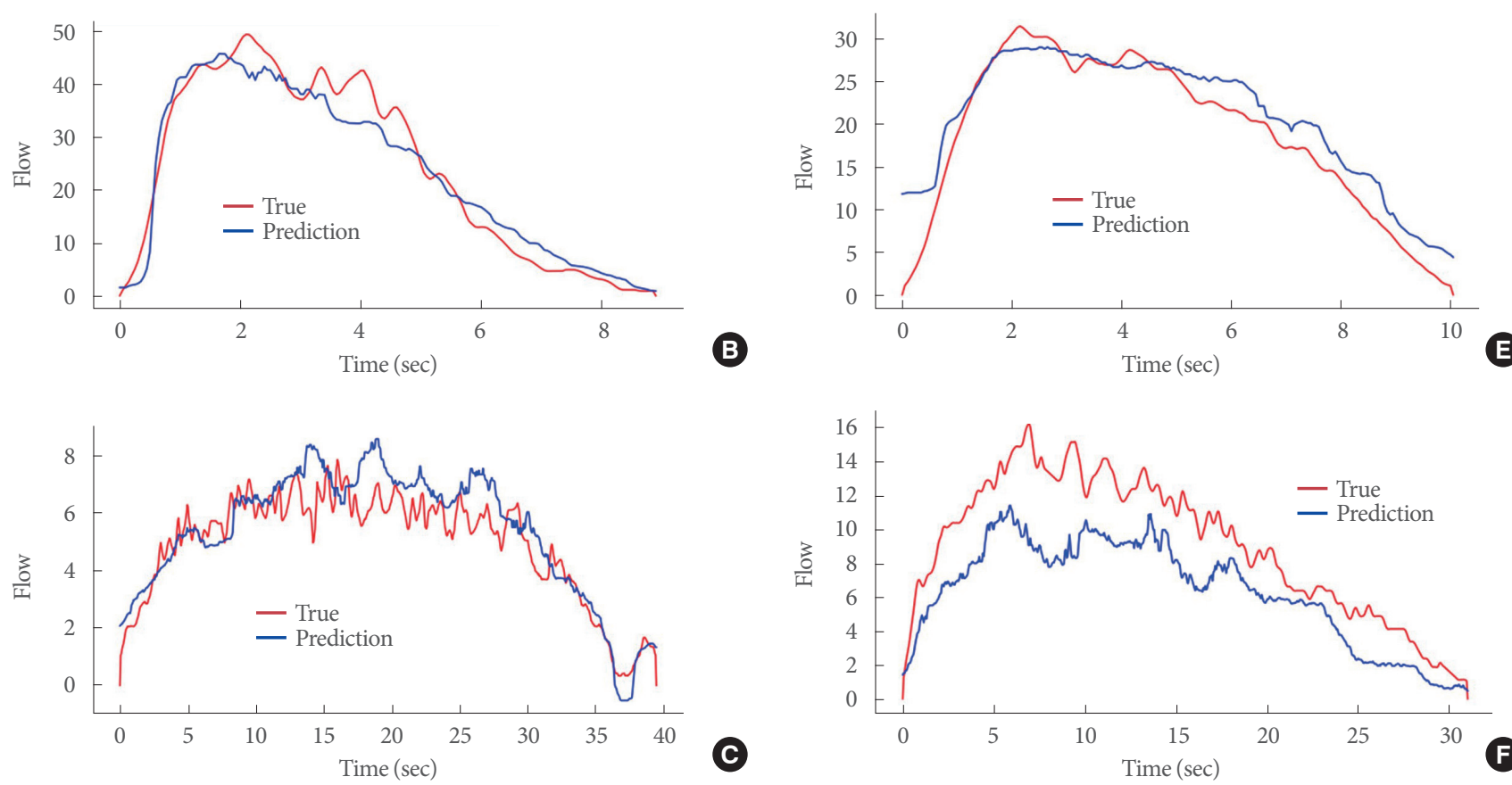

Fig. 3. Results of predicted flows in both sexes. (A-C) Examples of results of predicted flows in male. Overall flow patterns are well correlated. (D-F) Examples of results of flow patterns in female. (F) An example of female patient who has a correlated flow pattern but lower maximum flow rate.

However, even in those cases, flow patterns correlated well. Flow pattern disagreement existed in 1 female patient. The uri- nary flow rate was extremely low and flat without a characteristic sound, resulting in the error in prediction model. For void- 
Table 2. Comparison of the result of conventional uroflowmetry and acoustic uroflowmetry

\begin{tabular}{llccc}
\hline Sex & \multicolumn{1}{c}{ Parameter } & Conventional uroflowmetry & Acoustic uroflowmetry & Pearson correlation coefficient \\
\hline Male & Voided volume $(\mathrm{mL})$ & 202.0 & 200.9 & 0.95 \\
& Maximum flow rate $(\mathrm{mL} / \mathrm{sec})^{*}$ & 18.4 & 15.6 & 0.88 \\
& Average flow rate $(\mathrm{mL} / \mathrm{sec})$ & 9.3 & 9.4 & 0.91 \\
Female & Voided volume $(\mathrm{mL})^{*}$ & 233.5 & 219.0 & 0.96 \\
& Maximum flow rate $(\mathrm{mL} / \mathrm{sec})^{*}$ & 25.3 & 21.9 & 0.78 \\
& Average flow rate $(\mathrm{mL} / \mathrm{sec})^{*}$ & 13.2 & 12.2 & 0.93 \\
\hline
\end{tabular}

Values are presented as mean.

${ }^{\star} \mathrm{P}<0.05$ by Student $\mathrm{t}$-test.

ing parameters, all 3 parameters of Qmax, Qavg, and voided volume showed very strong correlations with PCC of $0.88,0.91$, and 0.95 , respectively (Table 2). This was also true for female participants' Qavg $(\mathrm{PCC}=0.93)$, voided volume $(\mathrm{PCC}=0.96)$, and $\mathrm{Qmax}(\mathrm{PCC}=0.78)$. Qmax differed between the 2 methods in both sexes $(\mathrm{P}<0.05)$. There was a statistically significant difference among females of the mean voided volume and Qavg while this was not observed in males for the same parameters.

\section{DISCUSSION}

Uroflowmetry is a simple, noninvasive, and basic office tool used to evaluate voiding dysfunction. Until now, no commercially available or reliable sound-based uroflowmetry has been used in clinical practice. We found that this new acoustic uroflowmeter had strong correlations of Qmax, Qavg, and voided volume in both sexes, suggesting the possibility of this acoustic uroflowmetry as a novel diagnostic test replacing current uroflowmetry. Because accuracy and reliability are a basic and necessary component for diagnostic tests to be used in clinical practice, further improvement in modeling and a future larger scaled study is required.

Sound-based analysis of uroflowmetry has been tried previously. The technical concept of sonouroflowmetry was introduced in 2009 [3] and clinical trials of sonouroflowmetry in male and female patients were reported in 2015 and 2016, respectively [8,9]. In their study using 25 male volunteers, the flow pattern showed a good visual correlation between the 2 methods. However, Qmax showed a weak correlation (PCC = $0.38)$ and Qavg, voided volume showed moderate correlation ( $\mathrm{PCC}=0.57$ and $\mathrm{PCC}=0.68$, respectively) [8]. In female patients, Qmax showed a weak correlation between uroflowmetry and sonouroflowmetry $(\mathrm{PCC}=0.38)$ [9] after analyzing 183 re- cordings of 36 healthy female volunteers. In their study, voided volume showed a moderate correlation ( $\mathrm{PCC}=0.68$ ).

In 2018, unlike previous studies on acoustic uroflometry, we were able to demonstrate a strong correlation between uroflowmetry and acoustic uroflowmetry with respect to Qmax, Qavg, and voided volume [10]. Flow patterns were almost identical if there was no loud noise. Urinary flow rate matched well even in very low flow rates. In addition to the developing interests in digital and mobile healthcare, this test can revolutionize the landscape of urology clinic by eliminating the need to wait and void in the uroflowmetry room. The mobile acoustic uroflowmetry can be done at home before visiting the clinics and provide more data for the clinician. The usefulness of portable, home-based uroflowmetry had been reported previously with a hand-held portable device [11,12]. In contrast to the previous literature, we have demonstrated that uroflowmetry can be accomplished through a smartphone-based sound recording without a special uroflowmeter which can be expensive and unwieldy. This new technology has the potential to improve patient care while reducing health care costs and the need for additional unnecessary devices, as well as improve the efficiency of urology clinics by eliminating the need to perform time-consuming office-based tests.

This study has a few limitations. Although the correlation of urine flow rate and predicted flow rate is excellent, Qmax was statistically significantly lower than the true value in both sexes, While the numerical difference is small, this may affect the overall accuracy. A revision of the prediction model is underway to improve the accuracy. It is also important to determine whether there is a pattern of discordance between the officebased and home-acquired acoustic uroflowmetry. Presumably, subtle differences such as toilet shapes, sizes, and water volumes may cause differences in the uroflow parameters. It will be nec- 
essary to test this model in various toilet settings for future use. In conclusion, uroflowmetry using acoustic analysis is a feasible modality producing comparable results to contemporary office-based uroflowmetry. Although Qmax is slightly lower than the actual value, the flow pattern correlated well. Further sophistication in modeling is necessary and underway. This new technology has the potential to change the landscape of urologic practice and improve patient care. With an increasing interest in digital health care, this uroflowmetry can be a part of home-based urologic care.

\section{AUTHOR CONTRIBUTION STATEMENT}

- Conceptualization: YJL, $M M K, S L$

- Formal analysis: $Y J L$

- Investigation: $Y J L$

- Methodology: YJL, MMK, SL

- Project administration: YJL, SL

-Writing-original draft: $Y J L$

- Writing-review \& editing: $S H S, S L$

\section{ORCID}

$\begin{array}{ll}\text { Young Ju Lee } & 0000-0003-2336-8871 \\ \text { Sang Hun Song } & 0000-0003-4888-483 X \\ \text { Sangchul Lee } & 0000-0002-3665-8336\end{array}$

\section{REFERENCES}

1. Alothmany N, Mosli H, Shokoueinejad M, Alkashgari R, Chiang M, Webster JG. Critical review of uroflowmetry methods. J Med Biol Eng 2018;38:685-96.

2. Urodynamic testing. In: Abrams Paul, Cardozo L, Wagg A, Wein A, editors. Incontinence. 6th ed. Bristol (UK): International Continence
Society; 2017. p. 604

3. Hitt D, Zvarova K, Zvara P. Urinary flow measurements via acoustic signatures with application to telemedicine. In: 39th AIAA Fluid Dynamics Conference. Fluid Dynamics and Co-located Conferences; 2009 Jun 22-25; San Antonio (TX), USA. Reston (VA): American Institute of Aeronautics and Astronautics; 2009.

4. Zvarova K, Ursiny M, Giebink T, Liang K, Blaivas JG, Zvara P. Recording urinary flow and lower urinary tract symptoms using sonouroflowmetry. Can J Urol 2011;18:5689-94.

5. Wiens K, Green S, Grecov D. Novel optical uroflowmeter using image processing techniques. Measurement 2014;47:314-20.

6. Shokoueinejad M, Alkashgari R, Mosli HA, Alothmany N, Levin JM, Webster JG. Video voiding device for diagnosing lower urinary tract dysfunction in men. J Med Biol Eng 2017;37:474-83.

7. Abrams Paul, Cardozo L, Wagg A, Wein A, editors. Incontinence. 6th ed. Bristol (UK): International Continence Society; 2017.

8. Krhut J, Gartner M, Sykora R, Hurtik P, Burda M, Lunacek L, et al. Comparison between uroflowmetry and sonouroflowmetry in recording of urinary flow in healthy men. Int J Urol 2015;22:761-5.

9. Gartner M, Krhut J, Hurtik P, Burda M, Zvarova K, Zvara P. Evaluation of voiding parameters in healthy women using sound analysis. Low Urin Tract Symptoms 2018;10:12-6.

10. Song M, Song J, Jung J, Lee S, Lee YJ, editors. Comparison of soundbased urinary flow prediction methods and validation with a large clinical sample. In: 25th International Congress on Sound and Vibration 2018 (ICSV 25); 2018 Jul 8-12; Hiroshima, Japan. Auburn (AL): International Congress on Sound and Vibration; 2018.

11. De La Rosette JJ, Witjes WP, Debruyne FM, Kersten PL, Wijkstra H. Improved reliability of uroflowmetry investigations: results of a portable home-based uroflowmetry study. Br J Urol 1996;78:385-90.

12. Hirao Y, Torimoto K, Shinohara M, Masumori N, Takeda M, Ishizuka $\mathrm{O}$, et al., editors. A novel handheld home-uroflowmetry, P-Flowdiary (R) [abstract 412]. In: ICS 2020 Online. Bristol (UK): International Continence Society; 2020. 